\title{
New results on low energy exclusive hadronic final states from BABAR
}

\author{
J. William Gary ${ }^{1, \star}$ \\ ${ }^{1}$ Department of Physics and Astronomy, University of California, Riverside, CA 92521, USA
}

\begin{abstract}
The 3.6 standard deviation discrepancy between the standard model (SM) prediction for the muon anomalous magnetic moment $g_{\mu}-2$ and the corresponding experimental measurement is one of the most persistent and intriguing potential signals in particle physics for physics beyond the SM. The largest uncertainty in the SM prediction for $g_{\mu}-2$ arises from the uncertainty in the measured low energy inclusive $e^{+} e^{-} \rightarrow$ hadrons cross section. New results from the BABAR experiment at SLAC for the $e^{+} e^{-} \rightarrow \pi^{+} \pi^{-} \pi^{0} \pi^{0}$ and $e^{+} e^{-} \rightarrow K K \pi \pi$ cross sections are presented that significantly reduce this uncertainty. New BABAR results for other low energy exclusive hadronic processes are also discussed.
\end{abstract}

\section{Introduction}

The gyromagnetic ratio of the muon, $g_{\mu}$, specifies the relationship between the muon's spin and its magnetic moment. The Dirac equation predicts $g_{\mu}=2$ exactly. Radiative corrections alter this prediction to $g_{\mu}=2\left(1+a_{\mu}\right)$. The term $a_{\mu}=\left(g_{\mu}-2\right) / 2$ is known as the anomalous moment of the muon, or, for brevity, the "muon anomaly." The muon anomaly is much more sensitive to virtual heavy particle production in loops than the corresponding electron anomaly because the relative contributions of the virtual terms scale like $\left(m_{\mu} / m_{e}\right)^{2} \approx 43,000$, where $m_{\ell}$ is the mass of lepton $\ell$. Theory and experiment agree to high precision for the electron anomaly. However, for the muon anomaly, there is a tension between theory and experiment of about 3.6 standard deviations (see, for example, Ref. [1]). This long-standing discrepancy is considered to be one of the most intriguing and potentially most significant indications for physics beyond the standard model (SM).

In the SM the muon anomaly is expressed as $a_{\mu}=a_{\mu}^{Q E D}+a_{\mu}^{E W}+a_{\mu}^{\text {had,LO}}+a_{\mu}^{\text {had,HO}}+a_{\mu}^{\text {had,LBLs }}$, where " $Q E D$ ", " $E W$ ", "had, $L O$ ", " $h a d, H O$ ", and "had, $L B L s$ " refer to the contributions from electromagnetic, weak, leading-order hadronic, higher-order hadronic, and hadronic light-by-light scattering terms, respectively. Example Feynman diagrams are shown in Fig. 1. Table 1 presents a summary of the relative size of the different terms, and their uncertainties. The electromagnetic term $a_{\mu}^{Q E D}$ is by far the largest term but has a small uncertainty. The second largest term, $a_{\mu}^{\text {had, } L O}$, from the leadingorder hadronic contribution, has, in contrast, a large uncertainty. Summing the terms yields a total SM prediction of $a_{\mu}^{S M}=(116591802 \pm 49) \times 10^{-11}$ [1], which lies $(287 \pm 80) \times 10^{-11}$ below the measured value of $a_{\mu}^{\text {data }}=(116592091 \pm 63) \times 10^{-11}$ [3]. This difference corresponds to a discrepancy of about

\footnotetext{
^e-mail: bill.gary@ucr.edu
} 

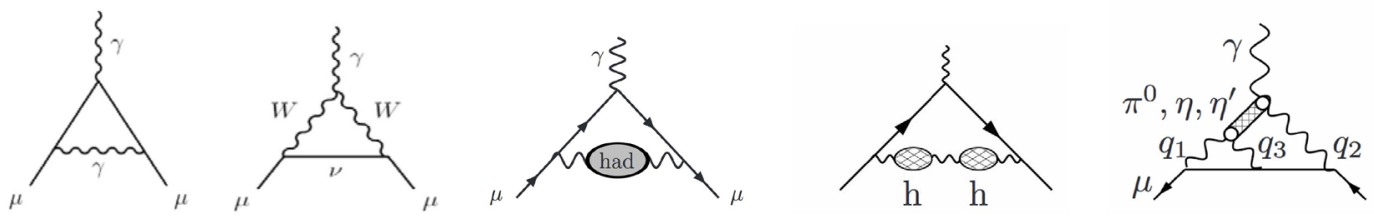

Figure 1. Feynman diagrams (from Ref. [2]) illustrating the contributions to the muon anomaly $a_{\mu}$ of (from left to right) electromagnetic, weak, leading-order hadronic, higher-order hadronic, and hadronic light-by-light scattering.

3.6 standard deviations. The overall uncertainty in the prediction, $49 \times 10^{-11}$, is dominated by the

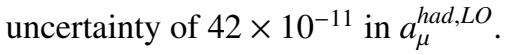

Table 1. SM results for the different contributions to the muon anomaly $a_{\mu}$ (from Ref. [1]).

\begin{tabular}{cc}
\hline$a_{\mu}$ term & SM prediction $\left(\times 10^{-11}\right)$ \\
\hline$a_{\mu}^{Q E D}$ & $116584718.951 \pm 0.080$ \\
$a_{\mu}^{E W}$ & $153.6 \pm 1.0$ \\
$a_{\mu}^{\text {had }, L O}$ & $6923 \pm 42$ \\
$a_{\mu}^{\text {had }, H O}$ & $-98.4 \pm 0.7$ \\
$a_{\mu}^{\text {had }, \text { } b L s}$ & $105 \pm 26$ \\
\hline$a_{\mu}^{\text {total }, S M}$ & $116591802 \pm 49$ \\
\hline
\end{tabular}

The longstanding tension in $a_{\mu}$ between experiment and theory could be an indication of new physics, such as from the supersymmetric process shown in Fig. 2 (left). The Muon $g-2$ experiment at Fermilab, with data collection scheduled to being in the Fall of 2017, expects to reduce the experimental uncertainty in $a_{\mu}$ by a factor of around 4 by 2019. The J-PARC E34 has a similar goal on a somewhat longer time scale. From the point of view of the SM prediction, the limiting factor is the uncertainty in the leading-order hadronic term $a_{\mu}^{\text {had,LO }}$.
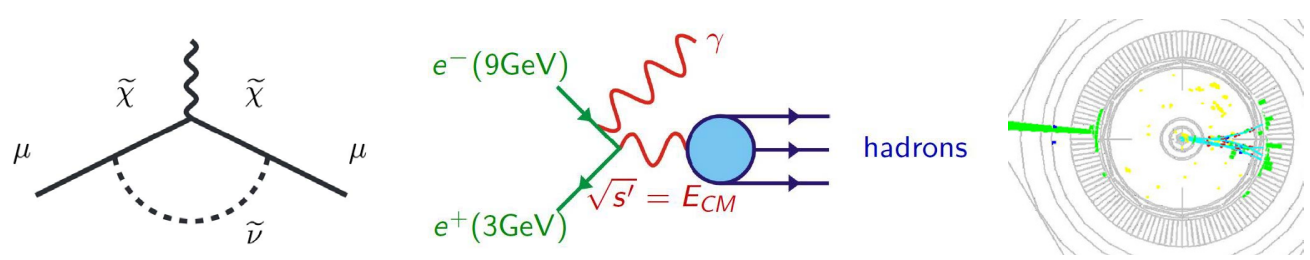

Figure 2. (left) Feynman diagram for a possible supersymmetric contribution to $a_{\mu}$; (center) illustration of the ISR process; (right) example display of an ISR event, in which a recoiling hadronic system (on the right of the display) is opposite to a high energy ISR photon.

The relevant energy scales are too low for $a_{\mu}^{h a d, L O}$ to be calculated perturbatively. Lattice calculations [4] are not yet sufficiently precise. Instead, the most precise result for $a_{\mu}^{\text {had, } L O}$ comes from low energy $e^{+} e^{-} \rightarrow$ hadrons data, using the optical theorem and the following dispersion integral (see, for 
example, Ref. [5]):

$$
a_{\mu}^{\text {had }, L O}=\left(\frac{m_{\mu}^{2}}{12 \pi^{3}}\right) \int_{m_{\pi}^{2}}^{\infty} \frac{\hat{K}(s)}{s} \sigma_{e^{+} e^{-} \rightarrow \text { hadrons }}(s) d s,
$$

where $s$ is the square of the center-of-mass $(\mathrm{CM})$ energy, $\hat{K}(s)$ is a kinematic factor with a magnitude on the order of unity, and $\sigma_{e^{+} e^{-} \rightarrow \text { hadrons }}$ is the inclusive $e^{+} e^{-} \rightarrow$ hadrons cross section. Because of the $1 / s$ factor, low energy contributions $(\sqrt{s}<2 \mathrm{GeV})$ dominate. At such low energies, the inclusive $e^{+} e^{-} \rightarrow$ hadrons cross section cannot be measured directly in a reliable manner because the simulations used to determine acceptance and reconstruction efficiencies are not reliable. Instead, one measures exclusive channels and sums their contributions, using isospin relations for unmeasured channels. Above around $\sqrt{s}=1.8 \mathrm{GeV}$ one can start to use perturbative QCD, and also to directly measure the inclusive cross section. The BABAR experiment at SLAC has a longstanding program to measure exclusive cross sections for all possible hadronic states below $\sqrt{s} \approx 2.0 \mathrm{GeV}$ and to thereby contribute to the determination of the SM prediction for $a_{\mu}$.

The BABAR experiment operated at the PEP-II asymmetric collider at SLAC between 1999 and 2008. Despite being shut down for almost 10 years, the data analysis is still active. There have been six BABAR papers submitted to journals so far in 2017, with a few others expected before the end of the year. The BABAR detector provided precise measurements of momentum and had excellent particle identification capabilities. Although it was primarily designed for the study of CP violation in $B$ meson decays, its general purpose design made it suitable for a wide variety of other studies, such as those discussed here. The analyses presented here make use of the final BABAR data set collected in the vicinity of the $\Upsilon(4 \mathrm{~S})$ meson, corresponding to around $470 \mathrm{fb}^{-1}$ of data at $\sqrt{s} \approx 10.6 \mathrm{GeV}$.

The measurement of low energy hadronic cross sections is performed with the initial-state radiation (ISR) method. Thus events are required to contain a high-energy photon emitted by the incoming electron or positron, as illustrated in Fig. 2 (center), lowering the effective CM energy of the hadronic system. In the BABAR studies, the photon with the highest energy is assumed to be the ISR photon. It must have a $\mathrm{CM}$ energy larger than $3 \mathrm{GeV}$. The ISR events are easily recognizable, as illustrated by the example event display in Fig. 2 (right), and have high event acceptance. The rate of final-state photon radiation is negligible. The ISR method allows BABAR to access a wide range of energies in a single experiment: from threshold to around $5 \mathrm{GeV}$. Point-to-point systematic uncertainties are essentially eliminated, unlike measurements based on an energy scan, in which the $e^{+}$and $e^{-}$beam energies are varied.

The study of intermediate resonance states in the hadronic systems is also important. Such studies shed light on the production process of hadrons and can be used to test theoretical models. From a practical point of view, knowledge of the intermediate resonance structure can be used to reduce systematic uncertainties in the acceptance and reconstruction efficiencies, since these terms depend on the intermediate state. For example, the angular distribution of particles depends on the intermediate state. Results from measurements of the intermediate resonance structure can be incorporated into Monte Carlo simulations, leading to a reduction in the uncertainties.

$2 e^{+} e^{-} \rightarrow \pi^{+} \pi^{-} \pi^{0} \pi^{0}$

The $e^{+} e^{-} \rightarrow \pi^{+} \pi^{-} \pi^{0} \pi^{0}$ cross section is one of the least known cross sections important for $a_{\mu}$. The final BABAR results on this channel, presented in Ref. [6], are now available. These results supersede preliminary BABAR results from 2007 that were based on only around half the final BABAR data set. Exactly two charged-particle tracks, an ISR photon candidate, and at least four other photon candidates are required. A kinematic fit is made to the $e^{+} e^{-} \rightarrow \pi^{+} \pi^{-} \pi^{0} \pi^{0}$ signal hypothesis, constraining two $2 \gamma$ combinations to the nominal $\pi^{0}$ mass. The combination of four photons that yields the smallest 
$\chi_{4 \pi \gamma}^{2}$ is retained. Signal candidates are required to have $\chi_{4 \pi \gamma}^{2}<30$. Background is subtracted using simulation normalized to data and a data sideband in $\chi_{4 \pi \gamma}^{2}$. Around 150000 signal events are obtained.
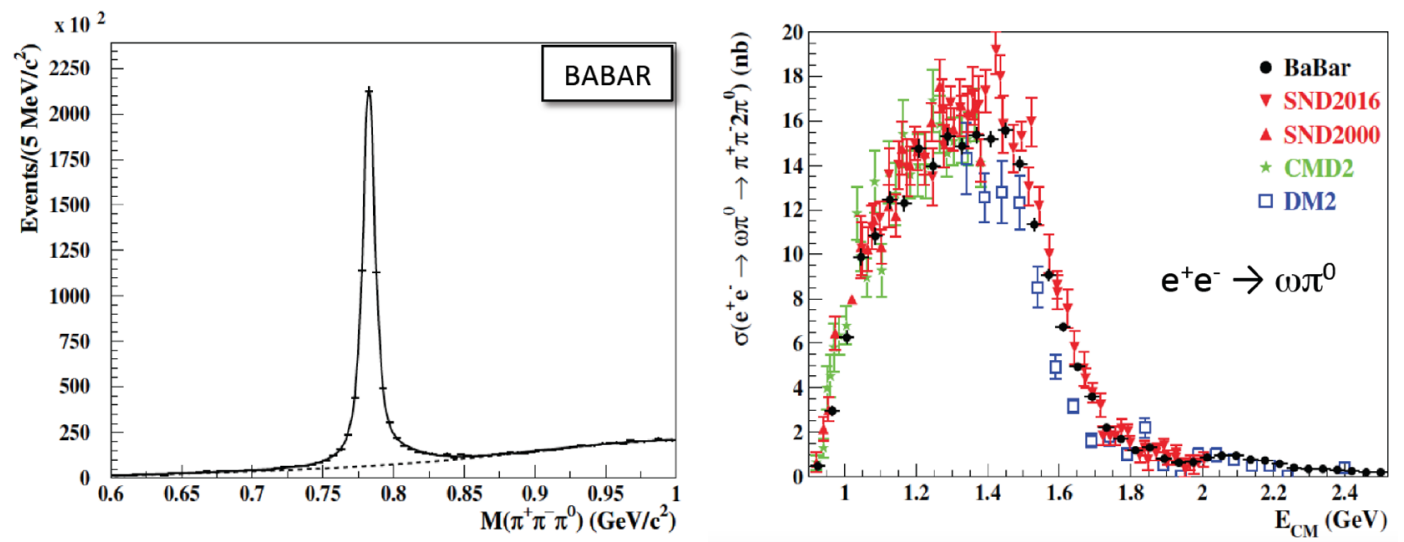

Figure 3. (left) The $\pi^{+} \pi^{-} \pi^{0}$ mass distribution in the selected sample of $e^{+} e^{-} \rightarrow \pi^{+} \pi^{-} \pi^{0} \pi^{0}$ events; (right) the $e^{+} e^{-} \rightarrow \omega \pi^{0}$ cross section.

A large fraction of the $e^{+} e^{-} \rightarrow \pi^{+} \pi^{-} \pi^{0} \pi^{0}$ cross section is found to be due to the $e^{+} e^{-} \rightarrow \omega \pi^{0}$ intermediate state with $\omega \rightarrow \pi^{+} \pi^{-} \pi^{0}$ decay. The $\pi^{+} \pi^{-} \pi^{0}$ mass distribution in the selected $e^{+} e^{-} \rightarrow$ $\pi^{+} \pi^{-} \pi^{0} \pi^{0}$ events is shown in Fig. 3 (left). A prominent peak at the $\omega$ meson mass is seen. Our measurement of the $e^{+} e^{-} \rightarrow \omega \pi^{0}$ cross section is shown in Fig. 3 (right). The BABAR results are seen to be more precise than previous measurements, cover a wider energy range, and resolve discrepancies between the measurements of other experiments.
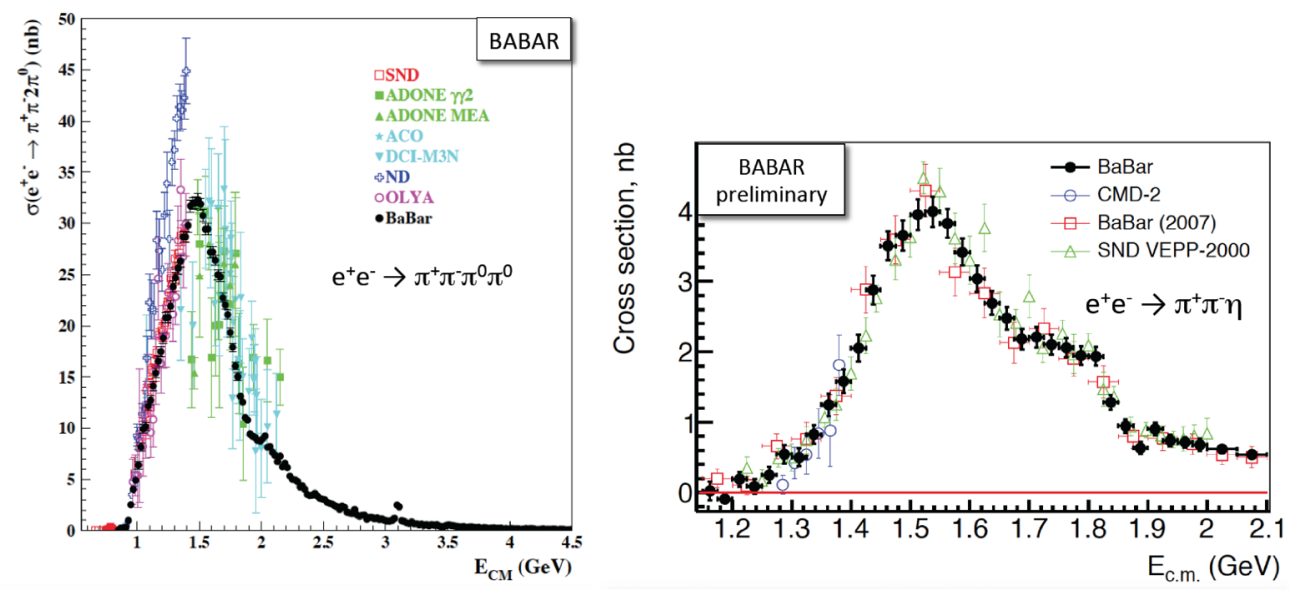

Figure 4. The (left) $e^{+} e^{-} \rightarrow \pi^{+} \pi^{-} \pi^{0} \pi^{0}$ and (right) $e^{+} e^{-} \rightarrow \pi^{+} \pi^{-} \eta$ cross sections.

Our result for the $e^{+} e^{-} \rightarrow \pi^{+} \pi^{-} \pi^{0} \pi^{0}$ cross section is shown in Fig. 4 (left). Again, the BABAR results are far more precise than previous results and cover a much wider energy range. 


\section{$3 e^{+} e^{-} \rightarrow \pi^{+} \pi^{-} \eta$ with $\eta \rightarrow \gamma \gamma$}

A second new result from BABAR, this one still preliminary and presently without a publicly available document, is a measurement of the $e^{+} e^{-} \rightarrow \pi^{+} \pi^{-} \eta$ cross section, based on $\eta \rightarrow \gamma \gamma$ decays. This result complements and improves the precision of the BABAR result for $e^{+} e^{-} \rightarrow \pi^{+} \pi^{-} \eta$ from 2007 [7], which was based on around half the final BABAR data set and utilized $\eta \rightarrow \pi^{+} \pi^{-} \pi^{0}$ decays. For the new result and for all other studies presented here, similar analysis techniques to those used to determine the $e^{+} e^{-} \rightarrow \pi^{+} \pi^{-} \pi^{0} \pi^{0}$ cross section, discussed above, are employed. The result for the $e^{+} e^{-} \rightarrow \pi^{+} \pi^{-} \eta$ cross section, based on a selected data sample of around 8000 signal event candidates, is shown in Fig. 4 (right). The measurements are seen to agree with previous results and to improve the precision, especially for higher values of $\sqrt{s}$.
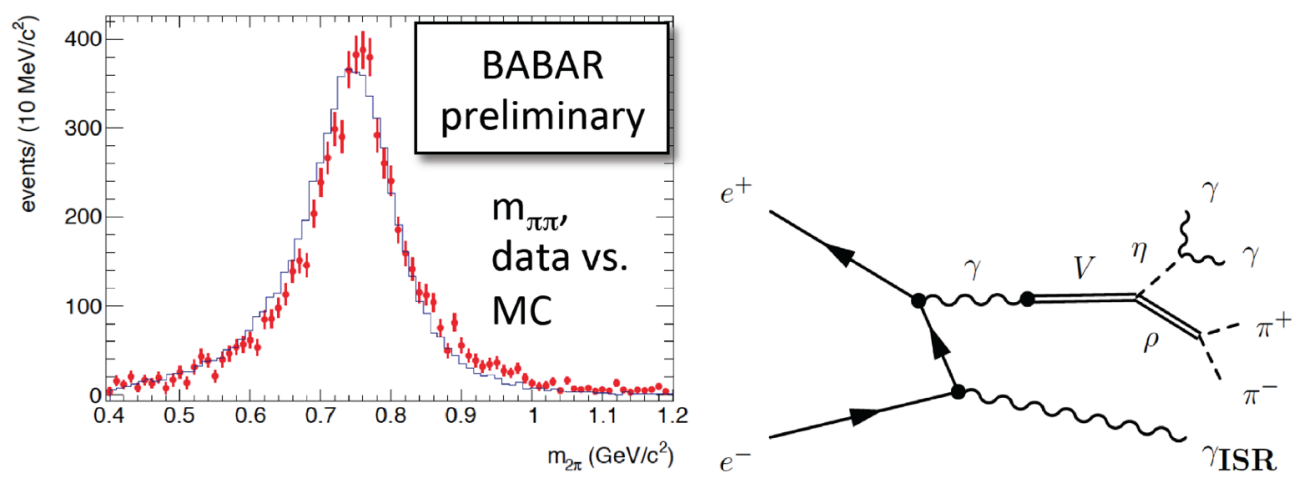

Figure 5. (left) The $\pi^{+} \pi^{-}$mass distribution in the selected $e^{+} e^{-} \rightarrow \pi^{+} \pi^{-} \eta$ events; (right) illustration of the vector meson dominance model for $e^{+} e^{-} \rightarrow \pi^{+} \pi^{-} \eta$.

We observe the $e^{+} e^{-} \rightarrow \pi^{+} \pi^{-} \eta$ reaction to be dominated by the $\rho(770) \eta$ intermediate state, as illustrated in Fig. 5 (left). However, the $e^{+} e^{-} \rightarrow \pi^{+} \pi^{-} \eta$ cross section has a complicated structure, as seen from Fig. 4 (right). We can use the vector meson dominance model, based on production through the $\rho$ (770) and higher mass $\rho$ states [see Fig. 5 (right)], to try to explain this structure. The coupling constants governing the decays are approximately real. Thus the phase differences between the different $\rho$ meson excitations are taken to be zero and $\pi$ only.

A model with two intermediate $\rho$ mesons, in which the $\rho(1450)$ is included with a phase difference of zero relative to the $\rho(770)$, does not fit the data and is discarded. However, a model in which these two $\rho$ mesons have a phase difference of $\pi$ yields the dashed curve (model 1) in Fig. 6, which is seen to describe the data up to $\sqrt{s} \approx 1.7 \mathrm{GeV}$. Adding a third resonance, the $\rho(1700)$, with either a relative phase of $\pi$ or zero yields the red-solid and dotted curves in Fig. 6 (models 2 and 3, respectively). These two models each describe the data up to approximately $1.9 \mathrm{GeV}$. Adding the $\rho(2150)$ meson (model 4) allows the data to be described up to $\sqrt{s} \approx 2.2 \mathrm{GeV}$, including the rather complicated structure in the 1.8 to $2.2 \mathrm{GeV}$ region. However, even for model 4 , the data lie above the prediction for $\sqrt{s}>2.3 \mathrm{GeV}$, implying that yet higher mass $\rho$ resonances may be relevant for the $e^{+} e^{-} \rightarrow \pi^{+} \pi^{-} \eta$ process. A summary of the different models tested is given in Table 2.

\section{$4 e^{+} e^{-} \rightarrow K_{S} K_{L} \pi^{0}, K_{S} K_{L} \eta$, and $K_{S} K_{L} \pi^{0} \pi^{0}$}

BABAR recently published results on the cross sections for the reactions $e^{+} e^{-} \rightarrow K_{S} K_{L} \pi^{0}, K_{S} K_{L} \eta$, and $K_{S} K_{L} \pi^{0} \pi^{0}$ [8]. These results are presented in this section. 

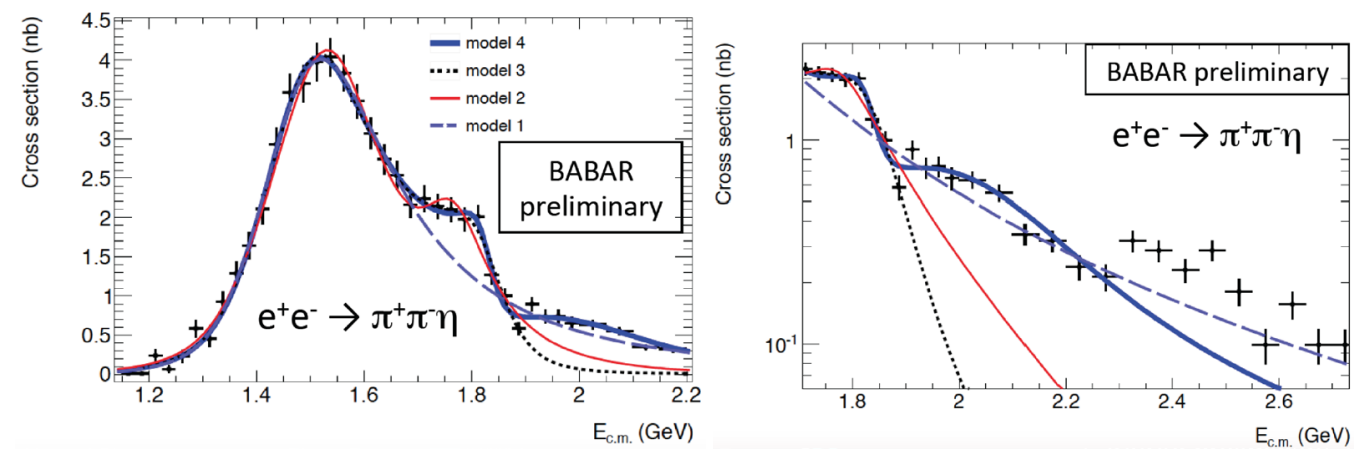

Figure 6. The measured $e^{+} e^{-} \rightarrow \pi^{+} \pi^{-} \eta$ cross section in comparison to predictions based on the vector meson dominance model: (left) for the full spectrum of measured results; (right) expanded view for $\sqrt{s}>1.7 \mathrm{GeV}$.

Table 2. Summary of different variants of the vector meson dominance model used to attempt to describe the $e^{+} e^{-} \rightarrow \pi^{+} \pi^{-} \eta$ cross section data.

\begin{tabular}{ccc}
\hline Model & Resonance model & Good fit for: \\
\hline 0 & $\rho(770)+\rho(1450)$ & Doesn't fit \\
1 & $\rho(770)-\rho(1450)$ & $\sqrt{s}<1.7 \mathrm{GeV}$ \\
2 & $\rho(770)-\rho(1450)-\rho(1700)$ & $\sqrt{s}<1.9 \mathrm{GeV}$ \\
3 & $\rho(770)-\rho(1450)+\rho(1700)$ & $\sqrt{s}<1.9 \mathrm{GeV}$ \\
4 & $\rho(770)-\rho(1450)+\rho(1700)+\rho(2150)$ & $\sqrt{s}<2.2 \mathrm{GeV}$ \\
\hline
\end{tabular}

Around 3700 signal events are obtained for $e^{+} e^{-} \rightarrow K_{S} K_{L} \pi^{0}$. The cross section is shown in Fig. 7 (left). This is the first measurement of this process. A peak is seen at the $J / \psi$ meson mass. This is the first observation of $J / \psi \rightarrow K_{S} K_{L} \pi^{0}$ decays. (For all other reactions discussed in the remainder of this report, the same statement applies: we obtain the first observation of $J / \psi$ decays to the presented channel.) The dominant intermediate state for $e^{+} e^{-} \rightarrow K_{S} K_{L} \pi^{0}$ is found to be $K^{*}(892) K$ [see Fig. 7 (right)]. Production through the $K^{*}(1430) K$ and $\phi\left(\rightarrow K_{L} K_{S}\right) \pi^{0}$ channels is also seen.

For the $e^{+} e^{-} \rightarrow K_{S} K_{L} \eta$ channel, we obtain 864 signal events. The measured cross section, which represents first measurement of this process, is shown in Fig. 8 (left). The process is dominated by the $e^{+} e^{-} \rightarrow \phi \eta$ intermediate resonant state, as illustrated in Fig. 8 (right).

Fig. 9 (left) shows the cross section for $e^{+} e^{-} \rightarrow K_{S} K_{L} \pi^{0} \pi^{0}$, based on a sample of 392 signal events. This is the first measurement of this process. The reaction exhibits clear evidence for the $K^{*}(892) K \pi$ intermediate resonant state [9 (right)], although in this case the nonresonant component also is substantial.

\section{$5 e^{+} e^{-} \rightarrow K_{S} K^{+} \pi^{-} \pi^{0}$ and $K_{S} K^{+} \pi^{-} \eta$}

In another recent publication [9], we present the first measurements of the $e^{+} e^{-} \rightarrow K_{S} K^{+} \pi^{-} \pi^{0}$ and $K_{S} K^{+} \pi^{-} \eta$ cross sections.

The result for the $e^{+} e^{-} \rightarrow K_{S} K^{+} \pi^{-} \pi^{0}$ channel, obtained from a selected sample of about 6400 signal events, is shown in Fig. 10 (left). A very large $J / \psi$ signal is seen. The $K^{*}(892) K \pi$ and $K_{S} K^{+} \rho(770)^{-}$intermediate resonant states are dominant [Fig. 10 (center) and (right)]. A clear 

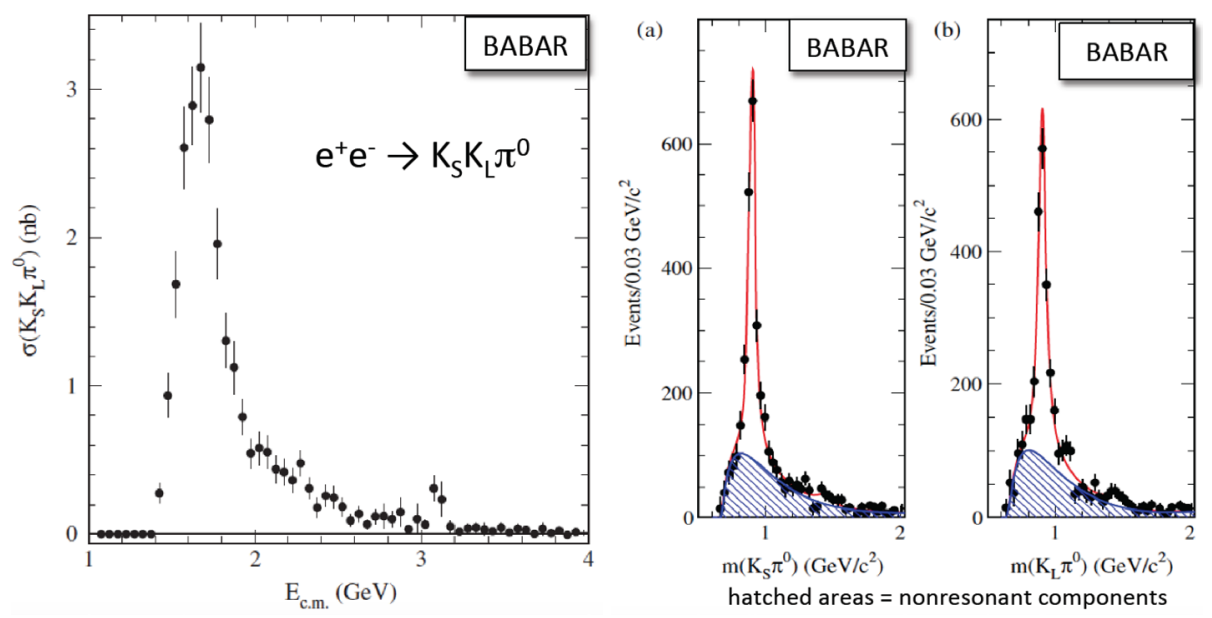

Figure 7. (left) The measured $e^{+} e^{-} \rightarrow K_{S} K_{L} \pi^{0}$ cross section; (right) the $K_{S} \pi^{0}$ and $K_{L} \pi^{0}$ mass distributions for the selected $e^{+} e^{-} \rightarrow K_{S} K_{L} \pi^{0}$ events.
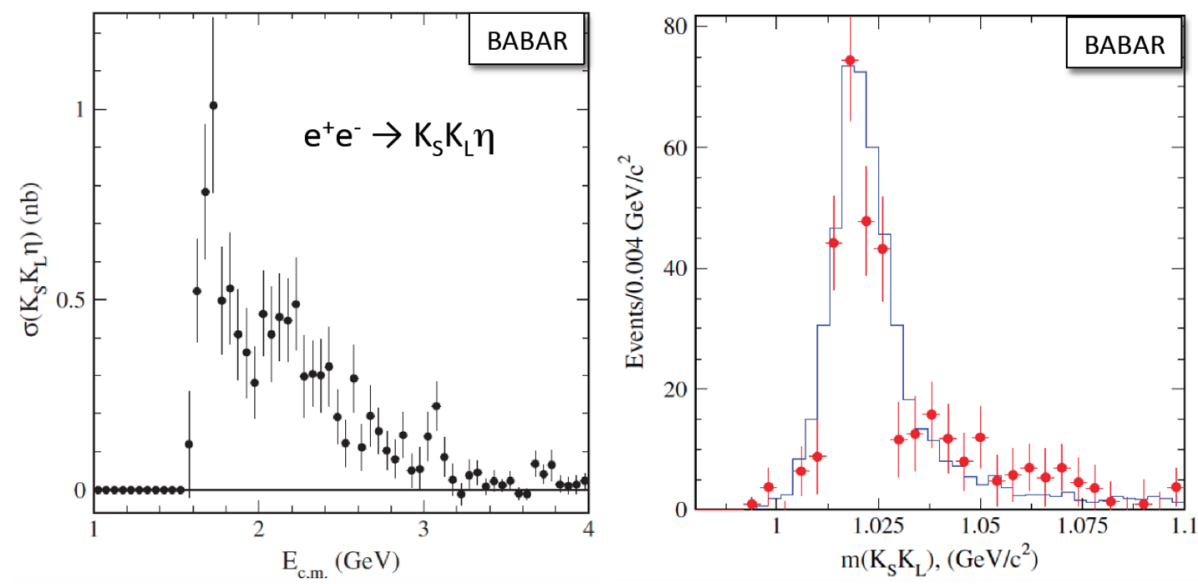

Figure 8. (left) The measured $e^{+} e^{-} \rightarrow K_{S} K_{L} \eta$ cross section; (right) the $K_{S} K_{L}$ mass distribution for the selected $e^{+} e^{-} \rightarrow K_{S} K_{L} \eta$ events.

$K^{*}(1430) K \pi$ signal is also seen. In addition, the double-resonant $K^{*}(892) K^{*}(892)$ intermediate channel is found to be responsible for around $15 \%$ of the total $e^{+} e^{-} \rightarrow K_{S} K^{+} \pi^{-} \pi^{0}$ cross section.

The result for the $e^{+} e^{-} \rightarrow K_{S} K^{+} \pi^{-} \eta$ cross section, based on a selected sample of 358 events, is shown in Fig. 11 (left). The results shown in Fig. 11 (center) and (right) demonstrate that much of this cross section arises from the resonant $e^{+} e^{-} \rightarrow K^{*}(892) K \eta$ process, particularly in the $K^{*}(892)^{ \pm} \rightarrow$ $K_{S} \pi^{ \pm}$channel. 

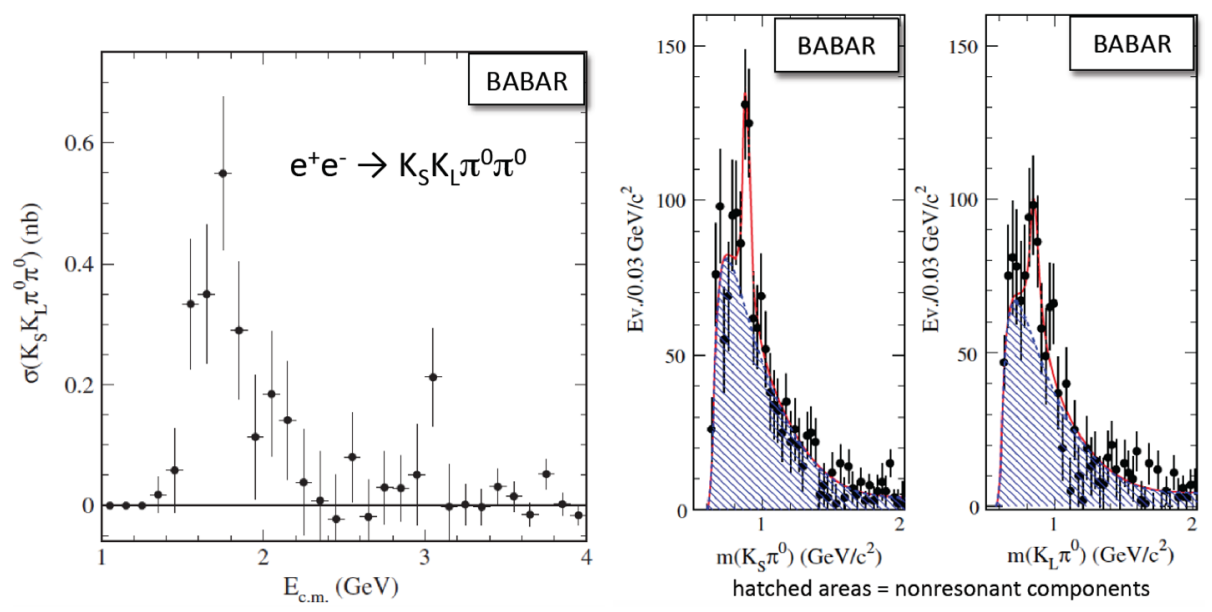

Figure 9. (left) The measured $e^{+} e^{-} \rightarrow K_{S} K_{L} \pi^{0} \pi^{0}$ cross section; (right) the $K_{S} \pi^{0}$ and $K_{L} \pi^{0}$ mass distributions for the selected $e^{+} e^{-} \rightarrow K_{S} K_{L} \pi^{0} \pi^{0}$ events.
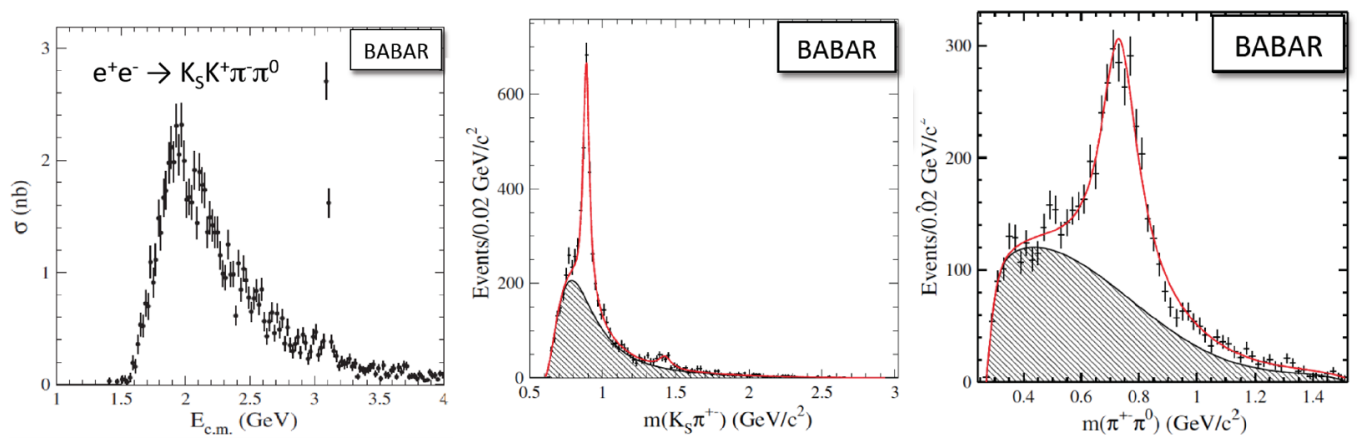

Figure 10. (left) The measured $e^{+} e^{-} \rightarrow K_{S} K^{+} \pi^{-} \pi^{0}$ cross section; the (center) $K_{S} \pi^{ \pm}$and (right) $\pi^{ \pm} \pi^{0}$ mass distributions for the selected $e^{+} e^{-} \rightarrow K_{S} K^{+} \pi^{-} \pi^{0}$ events.

\section{Implications for the muon anomaly $a_{\mu}$}

With respect to the muon anomaly $a_{\mu}$, the new BABAR $\pi^{+} \pi^{-} \pi^{0} \pi^{0}$ cross section measurements yield $a_{\mu}^{\pi^{+} \pi^{-} \pi^{0} \pi^{0}}=(179 \pm 6) \times 10^{-11}(3.2 \%$ precision $)$. The world average previously was $a_{\mu}^{\pi^{+} \pi^{-} \pi^{0} \pi^{0}}=(167 \pm$ $13) \times 10^{-11}$ (7.9\% precision). Thus the new BABAR $\pi^{+} \pi^{-} \pi^{0} \pi^{0}$ data reduce the uncertainty in $a_{\mu}^{\pi^{+} \pi^{-} \pi^{0} \pi^{0}}$ by more than a factor of 2 .

With the new results for $e^{+} e^{-} \rightarrow K_{S} K_{L} \pi^{0}, K_{S} K_{L} \pi^{0} \pi^{0}$, and $K_{S} K^{+} \pi^{-} \pi^{0}$, in conjunction with previous BABAR results published these past few years, BABAR has now measured all $e^{+} e^{-} \rightarrow K K \pi$ and $K K \pi \pi$ cross sections except those with a $K_{L} K_{L}$ pair. Thus, for the first time, the corresponding contributions to the muon anomaly, $a_{\mu}^{K K \pi}$ and $a_{\mu}^{K K \pi \pi}$, can be determined with no assumptions or reliance on isospin relations, except to assume the $K_{L} K_{L}$ rates to be the same as the $K_{S} K_{S}$ rates, which follows from CP symmetry. The $K K \pi$ channels comprise $12 \%$ of the total $e^{+} e^{-} \rightarrow$ hadrons cross section at $\sqrt{s} \approx 1.6 \mathrm{GeV}$. The $K K \pi \pi$ channels comprise $25 \%$ of the total $e^{+} e^{-} \rightarrow$ hadrons cross 

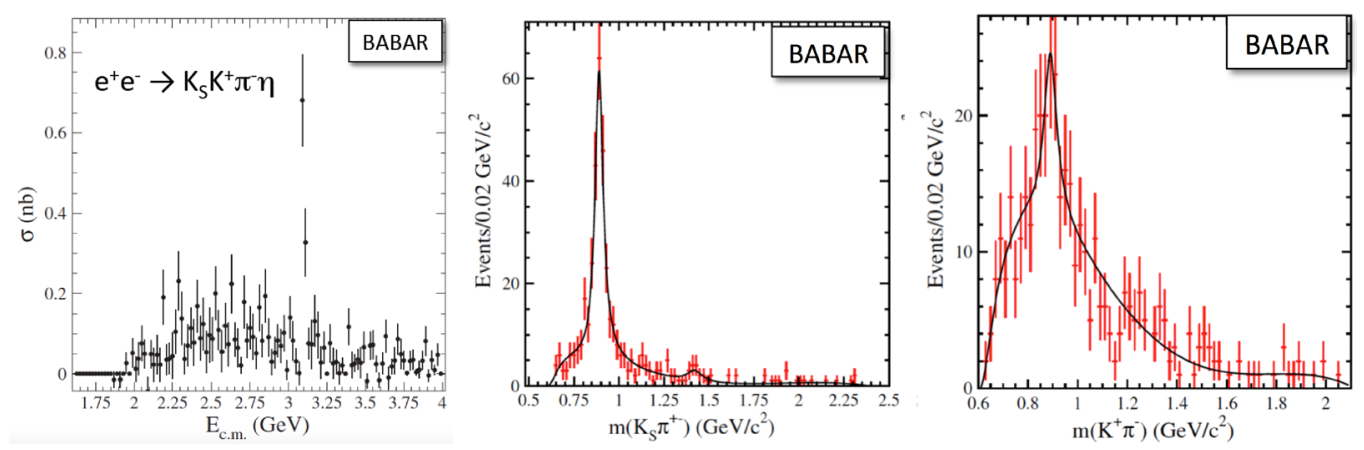

Figure 11. (left) The measured $e^{+} e^{-} \rightarrow K_{S} K^{+} \pi^{-} \eta$ cross section; the (center) $K_{S} \pi^{ \pm}$and (right) $K^{+} \pi^{-}$mass distributions for the selected $e^{+} e^{-} \rightarrow K_{S} K^{+} \pi^{-} \eta$ events.

section at $\sqrt{s} \approx 2 \mathrm{GeV}$. Beyond their importance for the muon anomaly, the new BABAR $K K \pi$ and $K K \pi \pi$ data will therefore be important in upcoming tests of the perturbative QCD prediction for the total $e^{+} e^{-} \rightarrow$ hadrons cross section at $\sqrt{s} \approx 2 \mathrm{GeV}$.

Using the new BABAR data, the result for $a_{\mu}^{K K \pi}$ is $(24.5 \pm 1.5) \times 10^{-11}$. However, this represents only a small improvement in precision compared to the previous result: $a_{\mu}^{K K \pi}=(23.9 \pm 1.6) \times 10^{-11}$. In contrast, for $a_{\mu}^{K K \pi \pi}$, the improvement in precision is very substantial: the result based on the new BABAR data is $(8.5 \pm 0.5) \times 10^{-11}(6 \%$ precision $)$ compared to the previous result $(13.5 \pm 3.9) \times 10^{-11}$ (30\% precision). The previous $a_{\mu}^{K K \pi \pi}$ result primarily relied on isospin relations.

In the big picture, the overall uncertainty in the muon anomaly $a_{\mu}$ is reduced from $42 \times 10^{-11}$ (Table 1) to $33 \times 10^{-11}$ [10], representing a $20 \%$ improvement, because of the new BABAR data.

\section{Summary}

Low energy $e^{+} e^{-} \rightarrow$ hadrons cross section data currently provide the most accurate prediction for

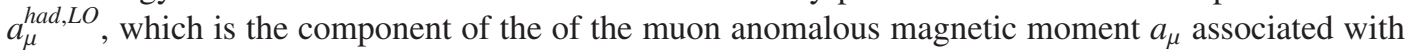
leading-order hadronic contributions. The uncertainty in $a_{\mu}^{\text {had,LO }}$ is the dominant uncertainty in the SM prediction for $a_{\mu}$. The $e^{+} e^{-} \rightarrow$ hadrons data are also important because they yield information on hadron dynamics and allow tests of QCD, such as for the perturbative QCD prediction for the inclusive $e^{+} e^{-} \rightarrow$ hadrons cross section. The data can also be used to provide (for example) the first observations of certain $J / \psi$ and $\psi(2 S)$ branching fractions.

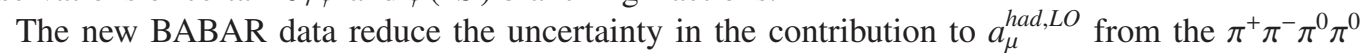
final state from 7 to $3 \%$. For $K K \pi \pi$ states, the uncertainty is reduced from 30 to $6 \%$. The overall uncertainty in $a_{\mu}^{h a d, L O}$ is reduced by about $20 \%$. Future progress in reducing the uncertainty in $a_{\mu}^{\text {had }, L O}$ will need to come from a reduction in the systematic uncertainty in the all-important $e^{+} e^{-} \rightarrow \pi^{+} \pi^{-}$ channel. New results on $e^{+} e^{-} \rightarrow \pi^{+} \pi^{-}$are expected from the BABAR and CMD3 experiments in the next year or so, which will hopefully resolve discrepancies that exist between current measurements.

\section{References}

[1] M. Davier, A. Hoecker, B, Malaescu, and Z. Zhang, Z. Phys. C 71, 1515 (2011).

[2] F. Jegerlehner and A. Nyffeler, Phys. Rept. 477, 1 (2009). 
[3] E821 Collaboration, G. W. Bennett et al., Phys. Rev. D 73, 072003 (2006).

[4] M. Della Morte, B. Jäger, A. Jüttner, and H. Wittig, JHEP 03, 055 (2012).

[5] Particle Data Group, C. Patrignani et al., Chin. Phys. C, 40, 100001 (2016) and 2017 update.

[6] BABAR Collaboration, J. P. Lees et al., arXiv:1709.01171, Submitted to Phys. Rev. D.

[7] BABAR Collaboration, J. P. Lees et al., Phys. Rev. D 76, 092005 (2007).

[8] BABAR Collaboration, J. P. Lees et al., Phys. Rev. D 95, 052001 (2017).

[9] BABAR Collaboration, J. P. Lees et al., Phys. Rev. D 95, 092005 (2017).

[10] M. Davier, arXiv:1612.02743, Nucl. Part. Phys. Proc. 287-288 (2017) 70. 